\title{
Yhwh y la conversión y la misión de su profeta. Análisis narrativo de 1 Re 19.
}

\author{
Yhwh and the Convertion and the Mission of \\ his Prophet. Narrative Analysis of 1 Re 19.
}

\author{
Federico Jiménez de Cisneros Ortiz \\ Pontifico Instituto Bíblico. Roma. Italia. \\ 8810@biblico.it \\ ID ORCID 0000-0002-2917-9118
}

\begin{abstract}
Resumen: El análisis narrativo se centra en el estudio de los tiempos de la narración y del discurso, la trama con sus distintas escenas, la perspectiva y los personajes. Frente a hipótesis que entienden 1 Re 19 como un rechazo a Elías, este análisis nos muestra que Yhwh lo acredita, resolviendo el problema de la soledad que sufre. Esto lo hace a través de la alimentación, la teofanía y la nueva misión que le encomienda.
\end{abstract}

Palabras clave: análisis narrativo, trama de resolución, teofanía, Elías, Yhwh, acreditación, vocación, misión.

\begin{abstract}
Narrative analysis focuses on the study of narrative and discourse tenses, the plot with its different scenes, the perspective and the characters. In contrast to hypotheses that understand 1Kings 19 as a rejection of Elijah, this analysis shows us that Yhwh accredits him, solving the problem of the loneliness he suffers. He does this through the feeding, the theophany and the new mission that he entrusts to him.
\end{abstract}

Keywords: narrative analysis, plot of resolution, theophany, Elijah, Yhwh, accreditation, vocation, mission.

\section{INTRODUCCIÓN}

La teofanía que Elías vivió en el monte Horeb y el resto de episodios que la acompañan conforman uno de los relatos acerca del cual más ríos de tinta se han vertido. Nos podemos fijar en muchos elementos sugerentes, como el mensajero de Yhwh que le proporciona alimento, la llamada a Eliseo o la famosa «voz de un susurro tenue» (1Re 19,12). Con todo, hay que preguntarse ¿cuál fue el problema que tenía antes? ¿Quién y cómo lo resolvió? ¿Qué sentido tiene lo que se nos narra que Dios hizo en este capítulo por Elías? ¿De qué manera las órdenes que le encomienda ayudan a Elías? El análisis narrativo, que incluye la escena previa y la posterior a la teofanía, o sea, todo el 
capítulo 19 del primer libro de los Reyes, enriquece la visión que se pueda tener de este pasaje y muestra, de una manera más completa, el sentido de este encuentro con Dios en la vida y misión del profeta. Veremos cómo todas las acciones que Dios va realizando (alimentación, diálogo, misión, sucesor) sirven para resolver el problema teológico que plantea Elías: su soledad en el culto a Yhwh y en la misión encomendada.

\section{ANÁLISIS TEXTUAL}

\subsection{Delimitación del texto y crítica textual}

La perícopa comienza en 1 Re 19,1. Es cierto que el wayyiqtol inicial indica continuidad en la cadena narrativa (y ciertamente la hay), aunque se observa un cambio de escena, preludiado por el trayecto hasta Yizreel (cf. 18,46); el milagro del Carmelo ha finalizado (cf. 18,40), y el narrador nos presenta una nueva etapa de la historia de Elías. En cuanto al final, la fórmula " $X+$ qatal" de 20,1 no deja lugar a duda sobre el comienzo de otro episodio. Esto es corroborado por el cambio de personajes y temática: desaparecen Elías y Eliseo y aparecen Ben Hadad y Ajab en el sitio de Samaría. Por lo tanto, la perícopa que nos ocupa es 1Re 19,1-21.

En cuanto a la crítica textual destaco las variantes más significativas, algunos de las cuales afectan de modo importante a la narración. Es necesaria la comparación con el texto de la LXX, que presenta frecuentes variaciones, pequeñas pero importantes. Hay que notar especialmente las de los vv.2-3, que orientan el relato de una manera específica. Entre las más importantes ${ }^{1}$ se encuentran:

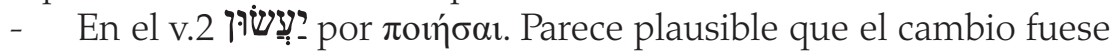
del singular (LXX) al plural (TM) para evitar que una idólatra jurase por el título divino. Puede entenderse que el pronombre $\mu \mathrm{ol}$ ha sido eliminado del TM, convirtiendo, como se verá más adelante, el juramento en una simple amenaza. Sin embargo, sigue estando presente en un buen número de manuscritos². El plus

1 Indico entre paréntesis la referencia del aparato crítico de la BHS cuando la hay. Hay otras variantes, que considero menores, las cuales introducen cambios ortográficos (4a), aclaraciones (1a, 9a), o directamente no cambian sustancialmente el sentido (19b).

2 Cf. BHS 2c: entre 16 y 60. 


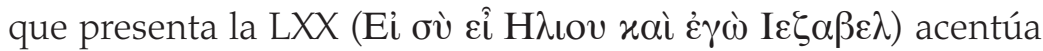
el antagonismo entre los personajes de Elías y Jezabel.

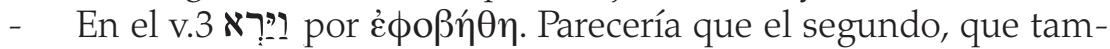
bién es seguido por algunos pocos manuscritos y la versión Siríaca y la Vulgata, tiene más sentido, aunque podría ser simplemente que Elías vio al mensajero, es decir, se dio cuenta de cómo estaba la situación y huyó ${ }^{3}$. Se puede entender que hubo una intencionalidad en TM para evitar presentar a un profeta de Yhwh presa del pánico ${ }^{4}$.

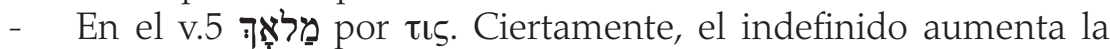
tensión narrativa ${ }^{5}$, aunque el efecto viene a ser el mismo, pues la identidad del mensajero continúa desconocida. Se puede entender que el autor tuvo una intencionalidad narrativa en el TM al usar el mismo término que ya ha aparecido en el v.2 referido a Jezabel: מֵֶֶ, el cual es, en ese versículo, innecesario. En cuanto a los términos para referirse a la planta bajo la cual durmió Elías, LXX traduce רתם por $\varrho \alpha \theta \mu$ (v.4) y $\phi v \tau o ́ v$ (v.5). Podría ser una nota explicativa que se introdujo en el lugar equivocado (v.5 del TM) y que LXX habría copiado 6 .

- En el v.8, el plus del TM הָאלהים parece plausible que sea una forma secundaria causada por un deseo de armonización con Ex $3,1^{7}$.

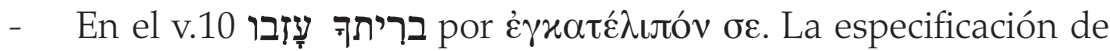
«tu Alianza» es seguramente un añadido deuteronomista ${ }^{8}$, pero no hay testimonios en hebreo de la variante griega.

- En el v.11 LXX tiene un plus: aúoıov. La consecuencia es que la teofanía será en el cuadragésimo primer día, y no en el

3 Cf. I. Provan, «An Ambivalent Hero: Elijah in Narrative-critical Perspective», Characters and Characterization in the Book of Kings (LHBOTS 670; London - New York - Oxford 2020) 135-151, 141.

4 Cf. P. Merlo, Re. Introduzione, traduzione e commento a cura di Paolo Merlo (Nuova versione della Bibbia dai testi antichi; Cinisello Balsamo 2020) IX, 209.

5 Cf. J. A. Montgomery, A Critical and Exegetical Commentary on the Books of Kings (The International Critical Commentary; Edinburgh 1960) 317.

6 Cf. P. Hugo, «Text and Literary History: the Case of 1 Kings 19 (MT and LXX)», Soundings in Kings. Perspectives and methods in contemporary scholarship (MN 2010) 15-33, 21.

7 Cf. Hugo, «Text and literary history», 20-21.

8 Cf. Hugo, «Text and literary history», 32. 
cuadragésimo como en el TM, del que parece más probable que se eliminase para que la teofanía tuviese lugar en ese día.

- En el v.12 LXX tiene el plus xảxeî xú oısos. Podría ser un añadido de carácter estilístico o explicativo", aunque también se puede vislumbrar una intención teológica.

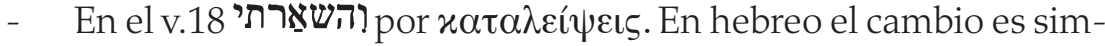
plemente por una «yod». La primera persona singular del TM acentúa la acción de Dios, presentando a un Elías más pasivo; LXX recalca lo contrario.

- En el v.20 el plus ולאממd TM es plausible que sea un añadido para dejar más completa la frase ${ }^{10}$.

A la luz de los casos estudiados, podemos concluir que la LXX tiene mejor estilo literario, más fluido y preciso ${ }^{11}$. Lo más importante es que la comparación de ambos textos aporta datos muy interesantes para el análisis narrativo. Según explica P. Hugo ${ }^{12}$, parece bastante plausible que el texto de LXX refleje una Vorlage más antigua que el proto-masorético o, al menos, que el TM fue editado con una clara intencionalidad narrativa, pues es más difícil que los cambios arriba señalados fuesen casuales ${ }^{13}$. Con todo, como suele ser habitual, no tenemos certeza completa. Los cambios en el TM dejan entrever una triple tendencia: «First, prophetic theology is modified accentuating the supremacy of the action of God over that of the prophet, the obedience of the prophet to the divine word, and the prophet's fidelity to the Torah. Second, the portrait of King Ahab is also modified in order to point out his guilt. Finally, the idolatry of the royal house is rendered more concrete and overwhelming ${ }^{14}$. Así, aunque aparentemente las diferencias sean menores, hay que hablar de distintas dinámicas narrativas fruto de distintas teologías.

9 «The Greek translator must have sensed the imbalance in the rhythm of the Hebrew text (...) It is just what we expected to hear from the narrator in the Hebrew Bible» C. E. MorRISON, «Elijah's Ineffable Experience», Bible Today 41 (2003) 355-358, 357.

10 Cf. Hugo, «Text and literary history», 19.

11 Cf. $1^{\mathrm{a}}, 1^{\mathrm{b}-\mathrm{b}}, 2^{\mathrm{b}}, 5^{\mathrm{a}-\mathrm{a}}$, el versículo 5 añade -BHS no lo recoge- un $x \alpha^{\prime}$ entre los dos imperativos del final, cosa que no sucede en v.7; $15^{\text {a }}$.

12 Cf. Hugo, «Text and literary history», 30.

13 Cf. A. Schenker, Älteste Textgeschichte der Königsbücher: Die hebräische Vorlage der ursprünglichen Septuaginta als älteste Textform der Königsbücher (OBO 199; Fribourg - Göttingen 2004) 27.

14 Hugo, «Text and literary history», 17. 


\subsection{Traducción, cuestiones filológicas y formas verbales más significativas}

(1) Narró Ajab a Jezabel todo lo que había hecho Elías y (todos los) que había matado a todos los profetas a cuchillo. (2) Envió Jezabel un mensajero a Elías diciendo:

- «Así hagan los dioses y así devuelvan; ciertamente mañana a estas horas trate ${ }^{15}$ tu vida como la vida de uno de ellos».

(3) Vio ${ }^{16}$ y se fue para [salvar] su vida, y llegó a Berseba de Judá y dejó a su criado allí. (4) Pero él anduvo por el desierto una jornada, llegó y se sentó debajo de una retama. Pidió a su vida morir y dijo:

- « Basta! Ahora Señor toma mi vida porque no soy mejor yo que mis padres».

(5) Se acostó y se durmió debajo de una retama, y he aquí que un mensajero ${ }^{17}$ comenzó a tocarle y le dijo:

- «Levántate, come».

(6) Echó un vistazo y vio en su cabecera una hogaza de brasas y un botijo de agua. Comió, bebión ${ }^{18}$, volvió y se acostó. (7) Volvió el ángel del Señor por segunda [vez], le tocó y le dijo:

- «Levántate, come, porque largo es tu camino».

(8) Se levantó, comió, bebió y anduvo con la fuerza de aquel alimento cuarenta días y cuarenta noches hasta la montaña de Dios, Horeb. (9) Llegó allí a la ${ }^{19}$ cueva, pernoctó allí y he aquí que la palabra del Señor [vino] a él y le dijo:

- «¿Qué a ti aquí, Elías?».

(10) Respondió:

15 Cf. L. Alonso SchöKel S. I., Diccionario bíblico hebreo-español (Madrid 1994) שים

16 El texto consonántico puede ser la forma defectiva del verbo temer, que concordaría con LXX.

17 Aunque se puede traducir por ángel, y luego (v.7) se sabrá que es el del Señor, prefiero el término «mensajero» para destacar el contraste con el del v.2.

18 Uso abusivo del wayyiqtol para acciones que de por sí son simultáneas, cf. P. JoÜON - T. MuRAOKA, Gramática del hebreo bíblico (Instrumentos para el estudio de la Biblia XVIII; Estella 2007) §118k. A partir de aquí citado como JM.

19 JM $\$ 137$ n3 sostiene que es una determinación imperfecta. Pero el cambio respecto a LXX invita a pensar que el redactor tuvo la intención de recalcar el paralelismo con Moisés. 
- «Ardo en celo joh Señor Dios de los ejércitos! $!^{20}$, porque han abandonado tu alianza los israelitas, tus altares han demolido y tus profetas han matado a espada; quedo yo solo y buscan mi vida para tomarla».

(11) Dijo [la palabra del Señor ${ }^{21}$ :

- «Sal y permanece en pie en la montaña ante el Señor, pues mira que el Señor está pasando».

Y un vendaval grande y fuerte descuajaba montañas y hacía trizas peñas ante el Señor, pero en el viento no estaba el Señor. Detrás ${ }^{22}$ del viento un terremoto, pero en el terremoto no estaba el Señor. (12) Detrás del terremoto un fuego, pero en el fuego no estaba el Señor. Detrás del fuego la voz de un susurro tenue ${ }^{23}$. (13) Cuando escuchó Elías, cubrió su rostro con su manto, salió y se puso de pie en la entrada de la cueva. Vino a él [la] voz y dijo: - «¿Qué a ti aquí, Elías?».

(14) Respondió:

- «Ardo en celo ¡oh Señor Dios de los ejércitos!, porque han abandonado tu alianza los israelitas, tus altares han demolido y tus profetas han matado a espada; quedo yo solo y buscan mi vida para tomarla».

(15) Le dijo el Señor:

- «Anda, deshaz ${ }^{24}$ tu camino hacia el desierto de Damasco, llega y unge a Jazael como rey de Aram. (16) A Jehú, hijo de Nimsí, unge como rey de Israel y a Eliseo, hijo de Safat, de Abel Mejolá unge como profeta sucesor de ti. (17) El que escape de la espada de Jazael [lo] matará Jehú, y el que escape de la espada de

Cf. M. DAHOOD, «Vocative Lamedh in 1 Kings 19,10.14», Bib 54 (1973) 407-408.

Por proximidad, el sujeto es «la palabra deYhwh», lo cual es frecuente en el ciclo de Elías (cf. 1Re 17,2.8; 18,1; 21,17.28).

22 La ausencia de verbos conjugados y el carácter descriptivo de la teofanía me invitan a traducir "detrás" en vez de "después", pues la noción de tiempo desaparece. Traduce con sentido temporal AlONSo ScHÖKEL, Diccionario bíblico hebreo-español,

23 Lit: «Voz de un silencio leve». Sigo la traducción propuesta en estas voces por Alonso ScHÖKel, Diccionario bíblico hebreo-español.

24 Lit: «vuelve»; LXX: «ve y vuelve a tu camino» N. Fernández Marcos - M. V. Spottorno Díaz-Caro - J. M. Cañas Reíllo (ed.), La Biblia griega Septuaginta. II Libros históricos (Biblioteca de estudios bíblicos 126; Salamanca 2008) 368. 
Jehú lo matará Eliseo ${ }^{25}$. (18) Voy a dejar ${ }^{26}$ un resto en Israel, siete mil, todas las rodillas que no se han doblado ante Baal y toda[s] la[s] boca[s] que no lo han besado».

(19) Se fue de allí y encontró a Eliseo, hijo de Safat; él estaba arando. Tenía doce yugadas y él estaba en la duodécima. Pasó Elías junto a él y lanzó su manto hacia él. (20) Dejó el ganado, corrió detrás de Elías y dijo: de ti».

- «Déjame besar a mi padre y a mi madre y que vaya detrás

Le respondió:

- «Ve, vuelve, porque ¿qué te he hecho a ti?».

(21) Volvió de [ir] detrás de él, tomó la yunta de ganado, la sacrificó y con los aperos del ganado (los) guisó la carne, [la] dio al pueblo y comieron. Se levantó, se fue detrás de Elías y le sirvió. Como en todas las narraciones, abundan las cadenas de wayyiqtol, que marcan la sucesión de los acontecimientos puntuales. Estos hilos narrativos son interrumpidos de diversas maneras. Normalmente por los diálogos de los personajes, en los que priman en este caso los imperativos (p. ej.: 4b, 15), y también por el mismo narrador, con una serie de frases nominales y existenciales de los vv. 11bc y 12 o con la fórmula"X+qatal" del v.4 (וּוּאיהלקה), que destaca al sujeto.

Otras formas verbales importantes son las que siguen. El v. 5

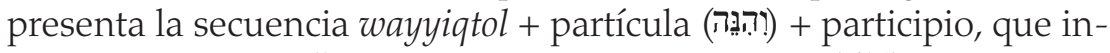
dica simultaneidad ${ }^{27}$. El waqataltí del inicio del v.17 (יָהיה:) indica el valor futuro de la frase que viene a continuación, que es una predicción; dos participios combinados con dos verbos en yiqtol. En el v. 18

25 Dos frases condicionales con simple yuxtaposición, cf. JM §167a, introducidas por חדיָיָ que se puede traducir por «y sucederá...» JM §119c. También se podrían considerar como temporales («cuando [alguno] escape... lo matará»); esto dependerá de la mayor certeza del hablante, que en este caso es Yhwh.

26 El waqataltí que sigue al participio con sentido de futuro, normalmente próximo, tiene también sentido de futuro, cf. JM §119n.

27 Estimo que en este caso no se le debe dar mucha importancia al aspecto durativo: se sobreentiende que le estuvo tocando... hasta que se despertó.“Here we have two simultaneous actions: the instantaneous one is expressed by the wayyiqtol, the durative by wĕhinnēh with the participle": L. SANTOPAOLO, "The "visionary" wĕhinnēh. The use of the construction wĕhinnēh with the participle in Dan 8", Cristianesimo Nella Storia 2 (2020) 619-632, 621-622. Esta misma partícula en los vv. 6.9.13 tiene valor perceptivo, y en el v.11 evidencial. Esto último es así porque desde el v.9 la perspectiva es la de Elías. 
encontramos de nuevo un waqataltí, que continúa la predicción; siguen dos verbos en qatal, con sentido de futuro perfecto ${ }^{28}$. El cohor-

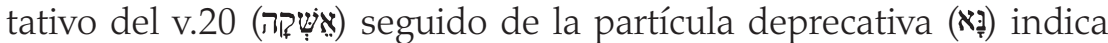

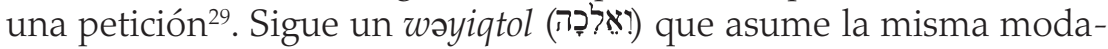
lidad. La respuesta con dos imperativos (שיכה) (שיב לה) debe entenderse no como una orden, sino con una modalidad permisiva.

Entre las frases con construcción más llamativa, destaco las siguientes.

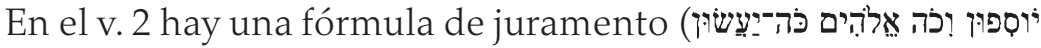

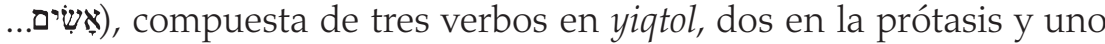
en la apódosis, en la que la partícula kî da el valor afirmativo ${ }^{30}$. El v. 4 finaliza con una frase nominal comparativa negativa de carácter

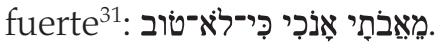

Pasando a los recursos sonoros y poéticos, un fenómeno curioso es la paronomasia de los vv. 10 y 14, causada por un infinito absoluto y un

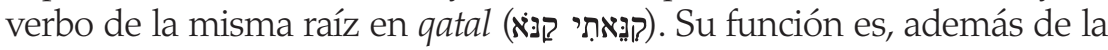
repetición sonora, acentuar la modalidad de la frase, en este caso afir-

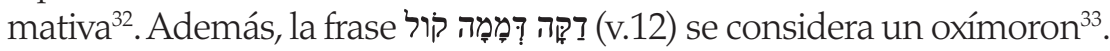

\section{ANÁLISIS NARRATIVO}

\subsection{Tiempo de la narración, tiempo del discurso}

Prácticamente toda la narración es presentada en la línea del relato, o sea, en el primer plano, con abundante uso del wayyiqtol narrativo que expresa acciones sucesivas. A veces se combina con qatal, casi siempre expresando acciones pretéritas al momento en que se habla. Los verbos en yiqtol o waqataltí sólo aparecen en los diálogos, donde no hay planos. Los cambios de ritmo vienen indicados principalmente por la ruptura de una cadena de wayyigtol, ya sea con un diálogo o con una frase nominal.

\footnotetext{
28 En proposiciones relativas que se refieren al pasado, cf. JM §112 i.

Cf. JM $\$ 114 \mathrm{~d}$.

Cf. JM §165 a-c.

Cf. JM $\S 160$ c.

Cf. JM $\$ 123$ de.

33 Cf. D. LawrIE, «Telling of(f) Prophets: Narrative Strategy in 1 Kings 18:1-19:18», JNSL 23.2 (1997) 163-180, 177.
} 
El narrador ha destacado tres escenas: la alimentación de Elías (4b-7); la teofanía en el Horeb con las órdenes divinas (9b-18); y la llamada a Eliseo (19-21). Se percibe claramente porque el ritmo de la narración se ralentiza por medio de los diálogos —en ellos el tiempo real y el narrado coinciden-, y de las pausas, en las que no hay noción de duración ${ }^{34}$ : la cadena de frases nominales y existenciales (vv. 11b.12) que forman la descripción de la teofanía, cuyo tiempo narrado es el más prolongado, y por lo tanto se concluye que es la más importante. Las tres escenas están unidas por dichas cadenas de wayyiqtol (vv. 3-4.8-9.19a).

También hay otros acentos en el texto. Ajab, en su relato a Jezabel (v.1), narra todo, pero destaca la matanza ${ }^{35}$. La huida hasta Berseba es veloz (v.3) ${ }^{36}$, pues las acciones se suceden una detrás de otra sin mediar pausa ninguna. En cambio, el trayecto desde Berseba hasta la retama tiene un ritmo más lento (v.4). El cambio es indicado por la

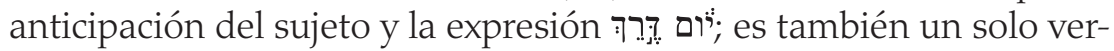
sículo, pero el tiempo real es menor. La llamada de Elías a Eliseo es apresurada (v.19), aunque después el ritmo se ralentiza para mostrar la radicalidad del seguimiento del que se deshace de sus elementos de trabajo compartiendo con los vecinos. Las repeticiones (vv.5.7 y 10.14) sirven para que no se pasen por alto los acontecimientos importantes: la alimentación y la pregunta divina.

Hay otra serie de momentos que el narrador simplemente ha obviado $^{37}$ : ¿Qué le respondió Jezabel a Ajab?, ¿no es plausible que le regañase? ${ }^{38}$, ¿por qué deja Elías a su criado y quién era?, ¿qué

34 Cf. J. L. SKA, «Nuestros padres nos contaron»: introducción al análisis de los relatos del Antiguo Testamento (Estella, Navarra 2012) 18.

35 Cf. C.Yebra Rovira, «Dios y Elías, su profeta, en 1 Reyes 19», EstBíb 64 (2006) 447$471,450$.

36 Si estaba en Yizreel fueron 5 ó 6 días de camino, para los que apenas dedica un versículo, cf. U. SIMON, Reading Prophetic Narratives. Translated from the Hebrew by Lenn J. Schramm (Indiana studies in biblical literature; Bloomington, IN 1997) 201.

37 A causa de la economía del relato, cf. SKA, Nuestros padres nos contaron, 17. O quizás debido al conocimiento de la audiencia, cf. T. S. HADJIEv, «Elijah's Alleged Megalomania: Reading Strategies for Composite Texts, with 1 Kings 19 as an Example», JSOT 39/4 (2015) 433-449, 443. El hecho de que haya hilos secundarios sueltos no quiere decir que el relato no tenga una unidad, como sostiene B. P. RoBINson, «Elijah at Horeb, 1 Kings 19:1-18: A Coherent Narrative?», RB 98/4 (1991) 513-536, 514-515.

38 Cf. SIMON, Reading prophetic narratives, 199. 
es lo que piensa el profeta? ${ }^{39}$, ¿dónde está la famosa retama?, ¿qué pasó durante los cuarenta días de viaje?, ¿quién le dice que debe ir al Horeb?, ¿o llega de casualidad? Además, en el v. 19 hay una laguna, pues no se narra las dos primeras unciones ${ }^{40}$. Más aún, la ejecución de la tercera, la de Eliseo, no se explicita. Otras carencias de información invitan al oyente/lector a suplir lo que falta y así involucrarse en la escena: cuando se le aparece el ángel ¿era ya de noche?; ¿en qué consiste «la voz de un susurro tenue»?, ¿cuál es el sentido de la pregunta que hace Elías en el v.20?

En los vv.1-9 la narración está salpicada de distintas intervenciones de los personajes (Jezabel, Elías y el ángel), sin que puede hablarse con propiedad de un diálogo, pues los discursos directos son meramente informativos y nunca hay una respuesta verbal por parte del interlocutor.

Hay dos momentos en los que aparece el segundo plano, que expresa una acción continua. En el v. 4 se corta la narración, pues Elías exhorta a Dios. Tras el paréntesis de 5a (se acuesta), al final del versículo recibe una orden, se entiende que como respuesta a su petición ${ }^{41}$. Justo aquí es donde se encuentra el segundo plano: la acción continua (participio) del ángel que le estaba tocando.

Algún comentarista considera que hay un intervalo que provoca suspense en el v. $9^{42}$, donde comienza el gran diálogo de Yhwh con Elías, que se subdivide en dos partes: los vv.9b-11a y los vv.13b-18. En medio se sitúan los fenómenos meteorológicos que preceden a la teofanía. Ambas partes comienzan exactamente de la misma forma, con la misma pregunta de Yhwh, con interrogación y vocativo (v. 9b:

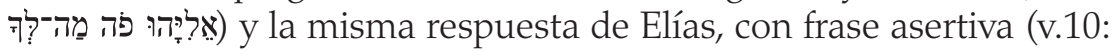

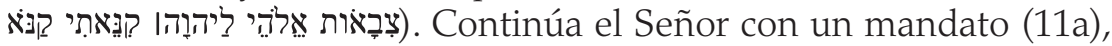
y el narrador retoma su función, dejando a Elías en la cueva (línea

39 Es cierto que conocemos su estado anímico por sus intervenciones, pues el narrador casi nunca nos revela sus sentimientos.

40 En realidad, no se explicita que Jazael sea ungido ni por Elías ni por Eliseo (cf. 2Re 8,7-14) y es Eliseo el que ordena a uno de sus propios discípulos que unja a Jehú (cf. 2Re 9,1-10). Surge la pregunta: ¿Por qué atribuir a Elías lo que Eliseo hará después? Más bien creo que hay que ver las acciones de Eliseo como confirmación de que él es el verdadero sucesor de Elías, el que continúa y completa su tarea.

41 Este esquema de narración seguido de diálogo (exhortativo) se encuentra también en vv. 6-7.

42 Cf. SIMON, Reading prophetic narratives, 205. 
de relato) mientras el Señor está pasando (participio como acción durativa: trasfondo). Los vv.13-14 son idénticos a los vv.9-10. Lo que cambia son las segundas respuestas de Yhwh.

Hay dos referencias a momentos pasados, en los vv. 1 y 10. En la primera el narrador dice que Ajab narra lo que había hecho Elías, fijándose en el asesinato de los profetas de Baal (cf. 1Re 18,40). En la segunda, que se refiere a una situación previa, Elías mismo (o el narrador por boca de Elías, si se prefiere), habla de tres acontecimientos que no aparecen explícitamente narrados en $1 R e$, aunque se encuentran diversas alusiones: el abandono de la alianza es denunciado con frecuencia (cf. 16,13.26), un altar había sido demolido (cf. 18,30), y algunos profetas del Señor fueron asesinados (cf. 18,4). Estas dos analepsis o recuerdos del pasado nos ayudan a situar este pasaje en el contexto de la historia de Elías, a la vez que aportan, o mejor, recuerdan, información en el momento oportuno para la intención del narrador: así se entiende por qué Elías es amenazado de muerte, huye y está cansado. De este modo, el lector está preparado para esperar la intervención de Dios, que ha conducido a Elías al Horeb.

En conclusión, pienso que, mediante las técnicas narrativas arriba indicadas, el interés del narrador ha sido destacar la teofanía ${ }^{43}$, dándole prioridad a la intervención de Dios, que es el que más habla y además lo hace en el centro del relato, en la escena principa ${ }^{44}$. Así, la atención no se desvía a las circunstancias adyacentes: la persecución o la situación religiosa y política.

\section{TRAMA}

\subsection{Aspectos generales}

El relato está ubicado durante el reinado de Ajab en Israel, o sea, durante el siglo IX a.C. Conociendo cuál es el marco histórico general, no contiene elementos que nos ayuden a esclarecer la fecha exacta en la que sucedieron los acontecimientos. El foco se ubica en la acción y el mensaje divino.

43 Cf. B. Brian, «Prophetic Concealment in a Biblical Type Scene», CBQ 64 (2002) 37-58, 49.

44 En los episodios anteriores Dios era el primero en intervenir, cf. MORRISON, «Elijah's Ineffable Experience», 356. Aquí va cobrando protagonismo conforme avanza el relato. 
La intriga de este episodio es unificada, aunque se encuadra en la intriga episódica del ciclo de Elías (1Re 17-2Re 2). Especial atención merecen $1 \operatorname{Re} 17$ y 18, que junto con este que nos ocupa se pueden considerar como un ciclo unitario ${ }^{45}$. Estos tres presentan una estructura con bastantes paralelos ${ }^{46}$. Hay un anuncio $(17,1 ; 18,1 ; 19,2)$; un viaje $(17,5 ; 18,2 ; 19,3)$, un doble encuentro (con la viuda y su hijo en 17,10.19; con Abdías y Ajab en 18,7.17; con el mensajero en 19,5.7); un milagro $(17,15.22 ; 18,38 ; 19,6)$ y una conversión $(17,24 ; 18,39$; $\left.19,21^{47}\right)$. El narrador muestra la teofanía como el culmen de esta primera parte de la actividad de Elías. Hay una clara progresión: la revelación de Dios va siendo cada vez más directa, tanto dentro del relato (ángel-voz-Yhwh) como en este ciclo (de la comida multiplicada a la revelación personal ${ }^{48}$. Además, en el lapso cambian los destinatarios de la experiencia: viuda pagana, pueblo de Israel y Elías. La relación de este con Eliseo va a ser ubicada en el contexto más amplio de la guerra entre Yhwh y Baal ${ }^{49}$.

\subsection{Análisis de la trama}

El punto de partida de la perícopa es la amenaza que sufre Elías, y el de llegada el tener un discípulo, por lo que ya no está solo. En medio está, como ya se ha dicho, el momento cumbre, el centro del relato: la teofanía y la misión. Sin la intervención divina ni se resuelve ni se entiende la vida del profeta ${ }^{50}$. Por primera vez en el ciclo de Elías el profeta se ha puesto en marcha por una causa humana, y no por

45 «Many of its episodes may originally have circulated independently; the signs of multiple authorship and redaction are clear. Yet, at the same time, the narrative as a whole has been carefully shaped both thematically and structurally», R. L. CoHN, «The Literary Logic of 1 Kings 17-19», JBL 101 (1982) 333-350, 334. Así, concluye afirmando: «the subordination of individual episodes, some of which may have circulated independently, into the present narrative context has created a whole greater than the sum of its parts (...) Their placement within the narrative as a whole determines the perspective in which the author meant them to be viewed» Ibidem, 349 .

Cf. CoHn, «The Literary Logic of 1 Kings 17-19», 343.

47 En cierta manera el seguimiento de Eliseo se puede considerar una conversión, no de la idolatría, sino un mayor reconocimiento de la divinidad de Yhwh.

48 Cf. CoHn, «The Literary Logic of 1 Kings 17-19», 347.

49 Cf. CoHN, «The Literary Logic of 1 Kings 17-19», 334.

50 Aunque en este capítulo no recurre a Dios, y cuando lo hace es para pedir la muerte, cf.Yebra Rovira, «Dios y Elías, su profeta, en 1 Reyes 19», 456. 
mandato divino ${ }^{51}$. Sin embargo, poco a poco el control va a ir pasando de Elías a Dios, para concluir con el profeta obedeciendo sus planes.

La persecución es la excusa del relato; lo importante es que crea la tensión necesaria para la trama y permite que inicie un viaje crucial para su vida y misión. Distintos motivos me llevan a afirmar esto. La amenaza que recibe en el v.2 no vuelve a ser mencionada al final de este relato ${ }^{52}$, ni siquiera en otras partes del libro. El hecho de que Jezabel envíe un mensajero indica, sin lugar a duda, que no quiere matarle, sino simplemente que huya ${ }^{53}$. Además, la ausencia de 'ל en la fórmula de juramento es otra señal de que no tiene intención de que sea efectivo ${ }^{54}$. La reacción es de interés y suspense esperando a ver en qué acaba la cosa, cómo concluye el viaje; por eso Jezabel desaparece, mientras que el lector acompaña a Elías en su periplo. Brian sostiene que el silencio de Dios y la retirada del profeta son elementos característicos de la escena profética ${ }^{55}$, y son necesarios para que tenga lugar la revelación. Es, al igual que en Ex 33, una trama con cuatro elementos: crisis, teofanía, nuevo comienzo y ejecución del plan divino ${ }^{56}$.

Por una parte, la trama es de resolución, porque el objetivo del relato es solucionar el problema que Elías le plantea a Yhwh: el profeta amenazado estaba solo. Sin embargo, no debe entenderse su soledad como una cuestión meramente física, sino sobre todo religiosa. Si él es el instrumento de Dios, ¿cómo seguir adelante con el plan divino para mantener el culto y enseñar al pueblo?, ¿qué debe hacer ahora que la situación sigue siendo complicada? Así, la soledad

51 Así 1Re 17,2-3.5; 18,1. Cf. Morrison, «Elijah's Ineffable Experience», 356. Aunque difiero de la siguiente opinión de este autor: «It was Jezebel's Word that brought him here [Horeb]». Ya explicaré cómo hay un giro en la primera escena, la de la retama, y así una novedad que podemos suponer que no entraba en los planes de Jezabel. Sin embargo, es cierto que esta vez lo ha movido la palabra de Jezabel y no la de Dios, cf. PROVAN, «An ambivalent Hero», 142.

52 Con lo que se descarta que esta sea la trama del relato, pues de ser así, iquedaría sin resolver! De hecho, en 21,20 Elías se vuelve a encontrar con Ajab y éste no le captura ni le hace nada, aunque sigan siendo enemigos.

53 Cf. R. E. Brown - J. A. Fitzmyer - R. E. Murphy (ed.), Comentario bíblico «San Jerónimo». Antiguo Testamento I (Madrid 1971) I, 532. Si no fuese así, ¡debería haber enviado directamente a un sicario!

54 Cf. R. J. Merecz, «Jezebel's Oath», Biblica 90 (2009) 257-259, 258.

55 Cf. BRIAN, «Prophetic Concealment», 37.58.

56 Cf. BRIAN, «Prophetic Concealment», 38. 
y la limitación de Elías es también soledad y limitación de Dios, cuyo instrumento y portavoz es Elías. Ya veremos que el narrador se sirve de este problema de la soledad de Elías para que la acción de Dios resuelva la trama revelándose y concretando su plan, pero de una manera que supera las expectativas de Elías, pues no obtiene un mero siervo, sino uno en calidad de sucesor. Concluye la narración en el v.21 con una peripeteia, ya anunciada en v.16, porque la soledad de Elías queda resuelta al llamar a Eliseo para que le siga.

Por otra parte, es cierto que hay un mayor conocimiento por parte de Elías (y del lector) acerca de Dios al final de relato, aunque no se debería hablar de anagnórisis, pues, aunque hay una información nueva (revelación de Dios en la brisa y proyecto divino de ungir), esta no resuelve el relato ${ }^{57}$. En este cambio de situación, tiene lugar un nuevo conocimiento acerca de Dios y su forma de revelarse ${ }^{58}$. Sin embargo, este nuevo conocimiento no es el objetivo final del relato, sino sólo un medio para llegar al objetivo, que es el arriba señalado: cambiar la situación de Elías. Así se da una «inclusio-inversio» ${ }^{59}$, pues el profeta vuelve a estar acompañado por alguien, pero no sólo eso, sino que esa persona es a la vez su sucesor, y las amenazas de Jezabel ya no van a guiar sus acciones. Por lo tanto, Elías es ratificado como profeta anteYhwh.

\subsection{Personajes, evolución de la trama y estudio de las escenas}

Los personajes humanos son Elías, el protagonista, Jezabel y Ajab, que sólo intervienen al principio (vv.1-2), y Eliseo, que aparece al final (vv.19-21). Los personajes divinos o asociados a la esfera divina son el mensajero de Yhwh (vv.5.7), la palabra de Yhwh (v.9), la voz (v.13) y el mismo Yhwh (vv.15-18). Pienso que al menos estos tres últimos, que aparecen en la misma escena, pueden ser considerados como un único personaje o personaje global, es decir, tres maneras de hablar que tiene el mismo protagonista.

57 Difiero de Vallançon, que afirma que en el TM la trama es de revelación, frente a LXX que la tendría de resolución, cf. H.VALLANÇON, Le développement des traditions sur Élie et l'histoire de la formation de la Bible (Études biblique. Nouvelle série; Leuven; Paris; Bristol, CT: Peeters 2019) LXXX, 49.

58 «Las repercusiones están frecuentemente al servicio de un cierto "despliegue" de la verdad, de la revelación de un determinado aspecto de Dios»: SKA, Nuestros padres nos contaron, 22.

59 SIMON, Reading prophetic narratives, 222. 
Por la descripción que Elías hace de su interior, «jbasta!» (v.4) y «ardo en celo» (vv. 10 y 14), el lector se siente atraído a solidarizarse con Elías ${ }^{60}$. La queja lo presenta débil, cansado, solitario. Pero es sobre todo el hecho de ser amenazado por los malvados (Ajab y Jezabel) lo que suscita en el oyente la identificación con este profeta fiel a Yhwh.

El v.1 constituye una exposición ${ }^{61}$, porque presenta los hechos y personajes indispensables (lo que había hecho Elías) y precede a la acción de la huida, ya que el juramento del v. 2 incorpora tensión y cambia el ritmo. Esto es así aún a pesar del hecho de que abunden los verbos de acción puntual (wayyiqtol y qatal).

He señalado antes los tres episodios que destaca el narrador, que corresponden con las tres escenas unidas entre sí que hacen avanzar la trama de una manera continua: alimentación de Elías (vv.48a), teofanía (9b-18) y llamada a Eliseo (19b-21). Corresponden a la preparación (comer para poder caminar), solución (diálogo con Dios y mandato divino) y resolución de la trama (vocación de Eliseo). A diferenciarlas nos ayudan los siguientes cambios: de acción (en la primera Elías duerme y come; en la segunda dialoga con Dios; en la tercera llama a Eliseo); de actores (ángel, Yhwh, Eliseo); de lugar (retama no identificada, monte Horeb, Abel Mejolá62); de ritmo (hay pausas en la narración: los viajes de Elías hacen la función de bisagra entre las diversas acciones de la narración). Cada escena debe ser considerada como preparación a la siguiente.

\section{A) Primera escena}

La primera escena, que se puede considerar típica de revelación ${ }^{63}$, es la complicación o intento de resolver el problema ${ }^{64}$. Así se mantiene la tensión necesaria, la cual se prolonga con el largo viaje - pues el lector se pregunta" ¿qué va a hacer Dios con Elías?"- y se va preparando el clímax, que es la teofanía del Horeb.

\footnotetext{
60 Aunque el hecho de degollar a los profetas de Baal, referido en v.1, repugne a nuestra mentalidad.

61 Cf.Yebra Rovira, «Dios y Elías, su profeta, en 1 Reyes 19», 449.

62 Se supone, aunque no se explicita.

63 Cf.Yebra Rovira, «Dios y Elías, su profeta, en 1 Reyes 19», 458.

64 Cf. SKA, Nuestros padres nos contaron, 27.
} 
Desde un punto de vista sincrónico ${ }^{65}$, tiene evidentes paralelos con la de Agar e Ismael (Gn 21,8-21): la huida en el mismo desierto, el peligro de muerte, la retama o arbusto; incluso Ajab y Jezabel son vistos como Abrahán y Sara ${ }^{66}$. Pero el más importante es la intervención de Dios, que produce el primer giro, pues la huida concluye y se comienza un nuevo viaje ${ }^{67}$; la intervención del ángel cambia la historia, porque el relato, que podría haber acabado allí, se desarrolla en otra dirección. Además hay paralelos con el Éxodo: Elías está en el desierto, y quiere morir allí, fuera de la tierra prometida, como murieron sus antepasados, porque verdaderamente no se considera mejor que ellos ${ }^{68}$.

Frente al mensajero de muerte de Jezabel, aparece otro mensajero en el v.5, aunque es uno de vida ${ }^{69}$ : ¿quién será? Resulta ser de origen divino (v.7), o sea, la respuesta de Dios que transforma la huida de muerte en peregrinación ${ }^{70}$. No sólo ha sido amenazado, sino que el mismo Elías desea morir. Creo que esto puede ser entendido como una muestra del sufrimiento, incluso de la desesperación, de la que él es preso a causa de la amenaza. Probablemente este sea el motivo por el que dejase a su criado en Berseba y se adentrase solitario en el desierto ${ }^{71}$. Pero quizás él quiso vivir la misma experiencia de inter-

65 Parece probable que $1 \operatorname{Re} 19$ fue puesto por escrito antes de Ex o Gn. Pero esto no es fundamental desde el punto de vista del análisis narrativo, sólo es crucial si queremos comprender cuáles fueron las primeras reacciones al escuchar este relato, y en nuestra situación, la teofanía de Ex enriquece nuestra perícopa.

66 Cf. M. Masson, Elie ou l'appel du silence (Parole présente; Paris 1992) 58.

67 Cf. A. J. Hauser, From Carmel to Horeb. Elijah in Crisis (Bible and Literature Series XIX; Sheffield 1990) 66. Junto con él, considero que el viaje al Horeb es cualitativamente diverso de la huida hasta la retama. Varios son los motivos: Elías toma un alimento especial; el viaje tiene una duración simbólica; a un lugar especial, cf. M. RoI, «1 Kings 19: A 'Departure on a Journey' Story», JSOT 37/1 (2012) 25-44, 37.

Cf. P. Zamora García, El libro de reyes, I. La fuerza de la narración (Nueva Biblia española; Estella 2011) 367.

69 Cf. Hauser, From Carmel to Horeb, 64.

70 Cf. Yebra Rovira, «Dios y Elías, su profeta, en 1 Reyes 19», 456. Es un personaje conector, que interviene en un momento puntual y desaparece, pero es imprescindible para la trama.

71 No solo la desesperación pudo haber sido un motivo: «Elijah decides to go off alone without provisions in order to seek death, leaving his servant behind - either so as not to have a witness to what he is to do; or (as De Vries, 235, suggests) as a sign that he no longer thinks of himself as a prophet; or because 
vención de Dios que Agar e Ismael ${ }^{72}$. De esta forma, habría sido un confiar en Dios, más que desesperar.

El alimento, que debe tomar dos veces, recuerda a los milagros de $1 \operatorname{Re} 17^{73}$. Ahora es el mismo Elías el que lo recibe: es una señal de que Dios sigue cuidando de él empezando por lo más básico y sencillo. Mediante la repetición del gesto de tocarle (dos veces), se intuye que el propósito del ángel es importante que se realice. Narrativamente hay una progresión, no es la mera repetición de un gesto ${ }^{74}$. Esta comida le permite peregrinar ${ }^{75}$ y muestra cómo Dios va preparándole; ha rechazado la muerte que pedía y ha reunido fuerzas ${ }^{76}$.

\section{B) Segunda escena}

La escena del Horeb es una teofanía. Si comparamos la perspectiva adoptada con otras teofanías ${ }^{77}$, se observa lo siguiente: hay una descripción panorámica del lugar, que es una cueva en Horeb (v.9a), pero no hay sumario proléptico, sino que inmediatamente, mediante וְה: (v.9b), se pasa a la focalización interna, gracias a la cual percibimos lo que él percibió ${ }^{78}$. Aunque con el diálogo del v.9c se vuelve a la focalización externa, el lector ya se ha quedado vinculado con Elías y su punto de vista.

$\mathrm{Al}$ igual que antes, aquí también hay paralelos más que evidentes con la teofanía de Moisés (Ex 33) ${ }^{79}$. Es fácilmente reconocible por la crisis de idolatría, el pensar en morir, el paso de Yhwh, la cueva, los cuarenta días, el diálogo, las nuevas órdenes, incluso el cubrirse con el manto (Ex 3). Sin embargo, hay algunas diferencias ${ }^{80}$ : Elías no intercede, sólo expresa su situación personal yYhwh le nombra un sucesor.

he is a poor hand at human relationships and, like Miss Garbo, prefers his own company» RoBINSON, «Elijah at Horeb», 517.

72 Masson, Elie ou l'appel du silence, 60.

73 Cf. Simon, Reading prophetic narratives, 204.

74 Cf. Hugo, «Text and literary history», 23.

75 El añadido הָתלהתים (v.8) del TM indica que no es un viaje cualquiera.

76 Cf. SimON, Reading prophetic narratives, 203.

77 Cf. SKA, Nuestros padres nos contaron, 74.

78 Se repite el mismo recurso usado en el v.6, cf. SKA, Nuestros padres nos contaron, 67.

79 Y también con Dt 4,24-28, donde un Dios celoso anuncia un exterminio casi completo, cf. Zamora García, El libro de reyes, I, 371.

80 Cf. BRIAN, «Prophetic Concealment», 56. 
En base a las distintas voces del diálogo, propongo esta estructura de la teofanía de Elías:

a) pregunta divina $(9 \mathrm{c})$.

b) respuesta de Elías (10),

c) respuesta deYhwh y acción divina descrita por el narrador (10-13a).

$\left.a^{\prime}\right)$ pregunta divina (13b).

b') respuesta de Elías (14).

c') respuesta de Yhwh (15-18).

Hay por lo tanto dos partes, introducidas por la misma pregunta de Dios, que a su vez recibe la misma respuesta de Elías. Esta repetición en paralelo sirve para destacar la situación lamentable de Elías, pero sobre todo para presentar lo importante: cómo Dios la resuelve ${ }^{81}$.

Se ha dicho que la queja de Elías es falsa, pues no corresponde con lo anteriormente narrado; que nos indica, además, que era soberbio $^{82}$, egoísta, desobediente ${ }^{83}$, cobarde $^{84}$, o que no sabe aprovechar las oportunidades que Dios le está dando cuando repite la pregunta $^{85}$. Esto provoca que Elías pierda credibilidad para sus detractores ${ }^{86}$, pues no es profeta modélico. Aunque es cierto que su figura, como cualquier otra, tiene luces y sombras ${ }^{87}$, voy a tratar de demostrar que es un profeta fiel a Dios.

Para tratar de explicar estas contradicciones, los críticos literarios ubican este capítulo antes del 18 o consideran los vv.11-14 como un

81 Digo que es lo importante porque la repetición suele servir para destacar lo crucial. Con todo, también puede ser que haya que prestar atención a lo que cambia, que sería lo novedoso, «variation in repetition is sometimes used to adumbrate not a feature of character but a development of plot»: R. Alter, The Art of Biblical Narrative (NY 1981) 100.

82 Cf. RoBinson, «Elijah at Horeb», 530.

83 Porque no cumple lo mandado, cf. P. J. KIssLING, Reliable Characters in the Primary History: Profiles of Moses, Joshua, Elijah and Elisha (JSOTS 224; Sheffield 1996) 144145. Sin embargo, ni le regañan, ni le castigan, ni Dios contradice su perspectiva, cf. HaDJIEV, «Elijah's Alleged Megalomania», 438.

84 Es cierto que en LXX, Elías sí tiene miedo, pero en el TM no se explicita tanto.

85 «The repetition does serve a literary purpose. After Elijah's unique glimpse of the depths of Yahweh's being, Yahweh has a right to expect a renewed commitment from Elijah. Instead Elijah's adamant refusal prompts Yahweh to relieve him on his post»: CoHN, «The Literary Logic of 1 Kings 17-19», 342-343.

86 Cf. Kissuing, Reliable Characters, 140.

87 Tengo que darle la razón a KissLing, Reliable Characters, 148. 
añadido y «3aßb-6,7-10,15-18» como el núcleo central del relato ${ }^{88}$. La amenaza de Jezabel (vv.1-3a) que serviría para conectar con el capítulo anterior, la teofanía (vv.11-14) y la vocación de Eliseo (vv.1921) serían añadidos posteriores. Siguiendo estas hipótesis, el relato original habría estado compuesto por la alimentación milagrosa, probablemente en un contexto de sequía, y un único diálogo con Yhwh seguido de la misión de ungir. De este modo, la trama de resolución queda empobrecida. Sin embargo, tomando el relato en su conjunto, éste es enriquecido y el análisis narrativo nos proporciona una posible solución a las contradicciones.

La intención del narrador al repetir la pregunta retórica ${ }^{89}$ de Dios y la respuesta de Elías es doble. Por un lado, muestra la realidad tal y como la ve el profeta, que hace un balance de la situación ${ }^{90}$ y nos descubre su interior: se siente profundamente solo, y esto lo expresa con mucha fuerza ${ }^{91}$. Por otro, da a Dios la ocasión más adecuada para hablar y manifestarse, y mostrar que, a pesar de la situación tan negativa, sigue adelante con su plan, asumiendo la situación ${ }^{92}$. Esta doble pregunta está en paralelo con la orden del ángel. Pareciese

88 Cf. J. KeInÄNEn, Traditions in Collision. A Literary and Redaction-critical Study on the Elijah Narratives 1 Kings 17-19 (Publications of the Finnish Exegetical Society 80; Helsinki 2001) 168.189. No entiendo cómo, si los vv.11-14 son un añadido postexílico, no se hubiese eliminado la referencia a los altares en plural (vv.10.14).

89 Al igual que Gn 4,9, cf. M. CogAN (ed.), 1 Kings: A New Translation with Introduction and Commentary (The Anchor Bible 10; New York 2001) 452. Si no, ¿qué sentido tiene que Dios le haga esa pregunta? Es cierto que no se explicita que el ángel le indique la meta de su peregrinación, aunque no se puede concluir que, después de la preparación con el pan y el agua, su llegada al Horeb fuese azarosa o casual, sino que tuvo una finalidad concreta. Hause considera la pregunta razonable porque no ha recibido información cf. From Carmel to Horeb, 67.

90 Se da la paradoja de que tras el Carmelo el pueblo ha abandonado la fe, sin embargo japenas ha habido tiempo!, cf. HADJIEV, «Elijah's Alleged Megalomania», 447. Aunque tal vez sea un sumario sin orden cronológico, cf. SimON, Reading prophetic narratives, 206.

91 «The repeated use of the first person in each of these three words, and the

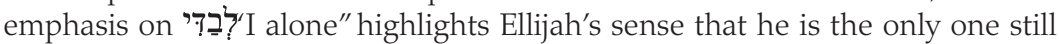
faithful to Yahweh»: Hauser, From Carmel to Horeb, 68. Esto podría entenderse más como una cualidad del profeta que como una situación temporal, pues Elías actuará en solitario en la viña de Nabot (1Re 21) y cuando anuncia el castigo a Ocozías (2Re 1). El relato resolverá su soledad con la sucesión, pero no con la compañía continua que cabría esperar.

92 Cf. SIMON, Reading prophetic narratives, 215. 
como si en ambos casos hubiesen hecho falta repeticiones para que el giro narrativo se produjese, para que Elías, y también el lector, se enterase y cambiase.

¿En qué consisten las dos intervenciones de Dios? En la primera hay una serie de fenómenos meteorológicos que se puede decir que constituyen el cortejo ${ }^{93}$ que precede a la divinidad, pero queda bien claro que no son ella. La repetición de la construcción יהוה... crea una gran tensión ${ }^{94}$. A esto se añade el v.13, donde Elías reconoce a Yhwh en la voz de un susurro tenue (la prueba es que se echa el manto por la cabeza y sale), y el lector le acompaña; Dios ha llegado ${ }^{95}$ y la tensión ¡se convierte en insoportable!

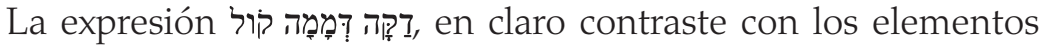
previos, es un oxímoron sin paralelos en la Biblia ${ }^{96}$, y tampoco es explicado por el narrador. El lenguaje, a través del sonido y la paradoja ${ }^{97}$, ayuda a expresar una experiencia inefable. Creo que aquí se cumple lo de que «menos es más». La enigmática frase del narrador recoge la experiencia de Elías, dejando abiertas para el lector infinidad de posibles interpretaciones, gracias a lo cual el segundo y más importante giro del relato aporta una gran riqueza ${ }^{98}$. El susurro tenue tiene una doble función:

If demamah is used in a particular way in Job 4,16 and $1 \mathrm{~K} 19,12$ to describe the reception of a revelation, a theophany, this is to be understood as a deliberate attempt to separate the Israelite concept of theophany from the religious ideas of the ancient Near East. At the same time, that which is

93 Cf. Cogan, 1 Kings, 453.

94 «Three consecutive repetitions, or three plus one, with some intensification or increment from one occurrence to the next, usually concluding either in a climax or a reversal»: Alter, The Art of Biblical Narrative, 96.

95 Cf. SKA, Nuestros padres nos contaron, 57. Ligado al punto de vista de Elías desde el comienzo de la teofanía, el lector no conoce directamente el pensamiento de Elías, pero reconoce a Dios porque Elías se cubre; esa acción es la que nos muestra su interior y el hecho de que es consciente de la presencia divina. Las traducciones más antiguas habrían hecho modificaciones; LXX añade xảxદî xú @ıos y la Peshitta lo asocia con Dt 4,12, para hacer el texto más inteligible a los lectores, cf. MORRISON, «Elijah's Ineffable Experience», 358.

96 Sl 107,29 y Job 4,16 tienen expresiones parecidas, pero no iguales.

97 «The phrase is rich in sound (note the chiastic series of consonants, q-d-m / $\mathrm{m}-\mathrm{d}-\mathrm{q}$ ) and paradoxical sense» J. T. WALsh, 1 Kings (Berit olam; Collegeville, MN 1996).

98 Cf. Morrison, «Elijah's Ineffable Experience», 355-358. 
totally imperceptible, intangible, invisible, and inaudible in the theophany is characterized most clearly. Theophany occurs when all tangible manifestations of power are out of the question, and yet it is just as immediately and intensively near as an unsuspected breath of air ${ }^{99}$.

Dios, que había demostrado ser más poderoso que Baal al dominar la lluvia (sequía) y mandar fuego del cielo, se revela ahora como no vinculado a estos fenómenos, libre también respecto a las exigencias que pudiesen tener sus seguidores acerca de las teofanías ${ }^{100}$. Él no es simplemente una divinidad más poderosa, sino que es cualitativamente distinto ${ }^{101}$. Sin embargo, cabe preguntarse: ¿por qué, al menos aparentemente, no hace efecto en Elías? La experiencia inefable de la voz, al menos según nuestra mentalidad, debería haberle cambiado a él y a su visión de la realidad. Sin embargo, el hecho de que Elías repita su respuesta no significa que no cambie; el panorama sigue siendo el mismo.

Lo primero que hay que destacar en la segunda intervención de Dios es que lo propio de Yhwh es hablar (frente al mudo Baal en 1Re 18,26). Sin embargo, ya no lo hace a través del ángel o de la voz, sino que él mismo se dirige a Elías; la comunicación es mucho más directa $^{102}$. Dios siempre había estado presente de alguna manera; ahora por fin ha llegado. Comienza dando órdenes muy concretas: volver y ungir a dos reyes y un profeta. La intervención de Dios afecta a la historia y a la política no sólo de Israel, sino también de otros pueblos que no son el suyo. Se añade un elemento más a esta nueva imagen de Dios. Algunos consideran que este cambio en el relato es señal de otra fuente, pero en el texto que nos ha llegado creo que cumple la función de mostrar cómo Dios resuelve los problemas y amplía los horizontes de su misión.

Las unciones reales son bien conocidas en la Escritura, no así las proféticas. Sería este un caso único en la Biblia, tanto por la unción como por escoger sucesor para el cargo profético ${ }^{103}$. Lo primero no llegará a realizarse, lo segundo sí (2Re 2). Algunos afirman que el

\footnotetext{
99 A. BAUMANN, «dāmāh II», TDOT II, IV.3.

100 «Yahweh's declining to be involve with the demonstrations of power in 19,1112 gives a clear message that Elijah cannot expect routinely to call on Yahweh's power in order to overwhelm his foes»: HAUSER, From Carmel to Horeb, 70.

101 Cf. LAWRIE, «Telling of(f) Prophets», 177.

102 Cf. SIMON, Reading prophetic narratives, 214.

103 Cf.Yebra Rovira, «Dios y Elías, su profeta, en 1 Reyes 19», 467.
} 
hecho de que Dios le ordene que unja a un profeta sucesor es señal de rechazo. Si verdaderamente Elías fuese válido, Dios no le hubiese buscado un sustituto. Sin embargo, creo que esto es una equivocación, por dos motivos. Primero porque la cuestión del sucesor resuelve, de una manera completa, la trama de este capítulo. No tendría mucho sentido que Dios interviniese para resolver un problema en favor de Elías y mediante la misma resolución el profeta fuese rechazado por Dios. Segundo porque tener una misión y un sucesor es señal de que Dios no ha rechazado a Elías ${ }^{104}$, sino que sigue confiando en su capacidad y en la tarea que todavía puede seguir realizando. Sí que es cierto que, en esta nueva situación, aunque él continuará, no va a ser el único protagonista. Tercero porque en la Biblia las teofanías son siempre para acreditar o ratificar a los que las reciben.

Continua Dios (vv.16-18) con una predicción que anuncia guerras y matanzas, pero en la que hay esperanza ${ }^{105}$, pues hay un resto, bastante más grande que lo que Elías pensaba, formado por los fieles a la alianza. El número de este grupo tiene un claro valor simbólico ${ }^{106}$. Aunque la guerra dure, la victoria es de Yhwh y Baal será derrotado.

Los paralelos, el privilegio de recibir una teofanía tan particular, la encomienda de una misión tan importante y el anuncio cargado de esperanza nos muestran que Elías es un interlocutor privilegiado ante Dios.

\section{C) Tercera escena}

La tercera escena constituye la resolución, pues se pone fin al problema inicial (manifestado en la segunda escena, en el v.10). Como conclusión, Eliseo le sirve, no como mero siervo, sino que se sobreentiende que como profeta ${ }^{107}$.

Lo primero que llama la atención son las elipsis de las unciones regias. Algunos sostienen que es una muestra más de la desobediencia de Elías o de su falta de fuerza ${ }^{108}$, pero en realidad el narrador ha

104 Como hizo por ejemplo con Saúl o Elí.

105 Cf. Yebra Rovira, «Dios y Elías, su profeta, en 1 Reyes 19», 464.

106 «Both seven and 1000 frequently serve as symbols of wholeness or completeness»: Hauser, From Carmel to Horeb, 77 nota 50.

107 Si Eliseo fuese un mero siervo, en muy poco habría cambiado la situación de Elías, que ya tenía uno, iy al cual él mismo despide!

108 Cf. Kissling, Reliable Characters, 110. O de que las consideró excesivamente revolucionarias, cf. HAuser, From Carmel to Horeb, 73. 
aplicado la ley de la economía narrativa para aquellos elementos que no pertenecen al objetivo de la trama.

Elías encuentra a Eliseo ${ }^{109}$, y el narrador aprovecha para situar la escena: estaba en un terreno muy grande, con una extensión de doce yugadas $^{110}$. En una rápida sucesión de acontecimientos, Elías lo llama de una manera muy particular ${ }^{111}$. Se inspira en la teofanía que ha vivido y hace lo propio, demostrando que algo sí ha aprendido: con el mismo manto con que se cubrió, cubre ahora a otro. Eliseo responde inmediatamente, pues abandona lo que estaba haciendo, corre y hace una petición (v.20). Se ha discutido mucho acerca del sentido de este requerimiento. Algunos opinan que es una dilación por parte de Eliseo ${ }^{112}$, otros que anuncia que Elías no estará del todo contento con su discípulo ${ }^{113}$ o con su pregunta. La enigmática respuesta de Elías es un eco ${ }^{114}$ de la pregunta divina (vv.9.13); por lo tanto, creo que puede ser entendida como una pregunta retórica, que apela a la libertad de la respuesta de Eliseo. Dicho esto, en cualquier caso hay que destacar la radicalidad del seguimiento del joven Eliseo y probablemente rico,

109 Aplicando la misma ley, tampoco se dice que llegase a Abel Mejolá o qué hizo para identificarlo, aunque probablemente lo conocería previamente, pues sus pueblos estaban cerca, cf. Zamora García, El libro de reyes, I, 374.

110 «1. f. Espacio de tierra de labor que puede arar una yunta en un día. 2. f. Medida agraria que equivale a 50 fanegas o a algo más de 32 hectáreas» R.- ASALE - RAE, «yugada | Diccionario de la lengua española» https://dle.rae.es/yugada [accedido septiembre 29, 2021].

111 Es cierto que no lo unge. Sin embargo, entiendo que sí se puede hablar de algún modo de consagración al echarle el manto encima. El manto, hecho con pelos

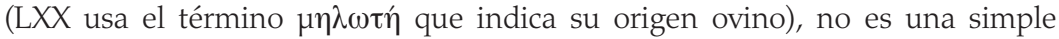
prenda. Suele ser el vestido oficial de determinados cargos (cf. 1Re 22,10; Jon 3,6; Est 6,8), en este caso de los profetas (cf. Zac 13,4). Además se le atribuyen poderes (cf. 2Re 2,8.13-14), cf. Yebra Rovira, «Dios y Elías, su profeta, en 1 Reyes 19», 467; Simon, Reading Prophetic Narratives, 219-220; MONTGOMERY, A critical and exegetical commentary, 316. Kissling sostiene que no hay verdadero traspaso de poderes, porque el manto vuelve a las manos de Elías, cf. KissLing, Reliable Characters, 124-125. Pero el mismo Eliseo lo recibe (cf. 2Re 2,13), como señal de la continuidad de la misión, y usa para realizar milagros, por lo que sí habrá continuidad.

112 Pero la pregunta es signo de reconocer la autoridad de Elías, cf. SIMON, Reading prophetic narratives, 221.

113 En 2 Re 2 puede dar la sensación de que Elías pone dificultades a Eliseo para seguirle, cf. SIMON, Reading prophetic narratives, 219.

114 Por la construcción לך, cf. N. Levine, «Twice as much of Your Spirit: Pattern, Parallel and Paronomasia in the Miracles of Elijah and Elisha», JSOT 85 (1999) 25-46, 43. 
el cual, sin haber visto ningún milagro ${ }^{115}$, por iniciativa propia sacrifica un par de bueyes (veinticuatro si tradujésemos yugada por yunta) y tras despedirse de sus padres, lo deja todo para comenzar a servir a Elías, rompiendo con la vida que llevaba ${ }^{116}$. De esta forma concluye el relato.

\section{MENSAJE Y CONCLUSIONES}

Ha habido una doble motivación a la hora de haber puesto por escrito este relato. En primer lugar, el resultado directo es una legitimación de la posición de Eliseo como profeta ${ }^{117}$, o al menos su presentación como auténtico sucesor o heredero de Elías, y por tanto verdadero profeta deYhwh ante Israel. Él, que más tarde lo llamará הָבְב אָבְ (2Re 2,12) es el personaje con el que Yhwh resolvió la soledad de Elías y la continuidad de su misión. Frente a lo que piensan varios autores, sostengo que Elías sale de esta prueba reforzado y acreditado. No es el final de su carrera o la última oportunidad que Dios le da ${ }^{118}$, sino que es un nuevo comienzo. Ciertamente, con un sucesor, lo cual ya nos hace pensar en que la misión se prolongará y otro cobrará protagonismo, pero será un sucesor suyo, al fin y al cabo. No hay un rechazo explícito por parte de Dios, sino una nueva y diferente misión. En segundo lugar, con unas consecuencias más amplias en la política interna de Israel, la unción de Jehú es directamente ordenada porYhwh y vinculada a Elías, el cual irá cobrando protagonismo en la tradición religiosa de Israel. Dándole un origen divino se legitima a la dinastía de Jehú, la cual eliminó completamente a la familia de Ajab, que era distinta de la suya (cf. 2Re 10,1-11), acabando también con la idolatría (cf. 2Re 10,18-27). El rey Jehú y su descendencia aparecerían queridos por Dios

115 Cf. SIMON, Reading prophetic narratives, 223.

116 Cf.Yebra Rovira, «Dios y Elías, su profeta, en 1 Reyes 19», 468.

117 «Elías y Eliseo poseen dos ciclos narrativos íntimamente entrelazados en la tradición, que los enmarca dentro del mismo movimiento e ímpetu profético. La garantía del primero está fundada en su experiencia sinaítica; para el segundo, dada su estrecha relación espiritual con Elías, basta probar su sucesión legítima, como en el caso típico Moisés-Josué. En ambos es Yahveh quien determina y confirma esa sucesión»: G. Del Olmo Lete, «La vocación de Eliseo», EstBíb 26 (1967) 287-293, 292.

118 Como sostiene Robinson, «Elijah at Horeb», 535. Si así fuese, ¿por qué recibe una misión? ¿por qué luego le serán encargada otras? ¿a qué viene esa ascensión en el carro de fuego? 
a causa de que persiguieron a los seguidores de Baal ${ }^{119}$.

Tras las apabullantes victorias de la sequía y el Carmelo, el relato nos ha presentado a un Elías débil en las dificultades ${ }^{120}$; no deja de ser paradójico que de una manera tan brusca y rápida se pase del éxtasis a la desesperación ${ }^{121}$. Esto, junto con las objeciones planteadas, han llevado a pensar que este capítulo fue escrito para dejar en mal lugar o ridiculizar a Elías. Sin embargo, hemos visto que las diversas acciones han buscado resolver su problema, mucho más que mostrarlo como alguien poco fiable. La dinámica no ha sido de la fama al rechazo por parte de Dios, sino de cómo, gracias a la acción continua deYhwh, Elías ha pasado de la soledad y la desesperación a tener un discípulo y ser ratificado por Dios.

La experiencia del Horeb debe ser para Elías lo que la del Carmelo fue para el pueblo ${ }^{122}$ : una ocasión para la renovación de la alianza. Diría que son tres las acciones que Dios hace o prepara en favor de Elías en este capítulo.

Primero lo alimenta, le vuelve a mostrar sus cuidados continuos y eficaces. Aunque él no se haya acordado de Dios en el primer momento, Yhwh sí que responde con prontitud y eficacia a sus peticiones. Esta intervención no sólo resuelve el problema concreto de Elías, sino que le prepara para algo mayor, que él no se podía imaginar. Después se revela de una manera especialísima y única; Yhwh se muestra como inabarcable e irreductible a los deseos o experiencias humanas. En tercer lugar, en esa teofanía se le encomienda una misión que tiene como objetivo asegurar la sucesión y la continuidad de su misión de profeta de Yhwh. Con la vocación de Eliseo se resuelve el problema de Elías y él ya está preparado para continuar con las misiones que Dios le ordene.

\footnotetext{
119 ¿O fue como consecuencia? Es decir, ¿persiguieron primero a los idólatras y luego los seguidores de Yhwh los apoyaron o primero se escribió el relato para justificar la unción de 2Re 9?

120 Provan, «An ambivalent Hero», 150-151.

121 Cf. Zamora García, El libro de reyes, I, 371.

122 Cf. Lawrie, «Telling of(f) Prophets», 174.
} 
